La revue La revue pour l'histoire du CNRS

7 | 2002

La biologie | Menaces sur les sciences sociales vers

1980

Entre biologistes, militaires et industriels :

l'introduction de la pénicilline en France à la

Libération

Jean-Paul Gaudillière

C OpenEdition

Journals

Édition électronique

URL : https://journals.openedition.org/histoire-cnrs/536

DOI : 10.4000/histoire-cnrs.536

ISSN : $1955-2408$

Éditeur

CNRS Éditions

Édition imprimée

Date de publication : 5 novembre 2002

ISBN : 978-2-271-06067-9

ISSN : $1298-9800$

Référence électronique

Jean-Paul Gaudillière, «Entre biologistes, militaires et industriels : l'introduction de la pénicilline en France à la Libération », La revue pour I'histoire du CNRS [En ligne], 7 | 2002, mis en ligne le 17 octobre 2006, consulté le 20 mai 2021. URL : http://journals.openedition.org/histoire-cnrs/536 ; DOI : https:// doi.org/10.4000/histoire-cnrs.536

Ce document a été généré automatiquement le 20 mai 2021.

Comité pour l'histoire du CNRS 


\title{
Entre biologistes, militaires et industriels : l'introduction de la pénicilline en France à la Libération
}

\author{
Jean-Paul Gaudillière
}

Introduction

1 En France, les premières années après la Seconde Guerre mondiale sont des années d'intense débat sur la « modernisation » du pays, ses dimensions politiques, économiques ou sociales. Les sciences n'échappent pas à ce mouvement. Dans ce domaine comme dans les autres, l'heure est au bilan critique de l'entre-deux-guerres, à la mise en accusation du retard accumulé, à la rédaction de toutes sortes de projets et programmes d'action' ${ }^{1}$.

2 Pour les sciences de la vie et de la santé, la seconde moitié des années 1940 débouche sur des changements institutionnels importants comme la réorganisation du CNRS, de l'Institut national d'hygiène (INH, fondé en 1942) ou l'adoption des ordonnances sur la sécurité sociale. Celles-ci font de l'État le premier entrepreneur de recherche, inaugurant une longue période de croissance des investissements ${ }^{2}$. Les transformations ne se limitent pas aux infrastructures; elles touchent également les objectifs et priorités d'investigation, conduisant à ce que l'on peut désigner comme une " biomédicalisation ». Cette dernière a pour caractéristique non pas le fait que l'on aurait commencé à considérer le laboratoire de biologie comme une source de savoirs utiles à la médecine (cette perspective était déjà au centre de la révolution bactériologique de la fin du XIX siècle), mais une transformation profonde des formes de leur mise en relation. La biomédecine des années 1945-1965 est ainsi marquée par le développement de la modélisation, par la mise en avant des molécules et macromolécules biologiques comme objets privilégiés d'étude du normal et du pathologique, par l'importance accordée à l'innovation chimiothérapeutique, et par le développement des procédures de jugement statistique des thérapies ${ }^{3}$.

3 Compte tenu des conditions de la mobilisation scientifique, du rôle dominant acquis par les États-Unis en matière technique, économique et politique, cette reconfiguration a 
eu lieu « au miroir de l'Amérique ». Du moins si l'on entend par « miroir » non pas un simple transfert ou une copie d'un modèle de recherche, mais le fait que les chercheurs français ont vu dans le complexe biomédical américain à la fois un horizon pour réfléchir la situation française, l'origine de nouveaux moyens et un repoussoir. Les biologistes et médecins français d'après 1945 ont imaginé leur Amérique. Ils l'ont régulièrement visitée. Ils en ont massivement mobilisé les ressources (argent, instruments ou résultats). Ils l'ont toujours fait en fonction de leurs propres perspectives. L'histoire des antibiotiques, de leur développement et production en masse, est emblématique de ce processus de biomédicalisation et de circulation transatlantique. Suivre l'introduction de la pénicilline dans la France de 1945-1946 permet d'explorer certaines des tensions entre les acteurs associés à l'édification de la nouvelle biomédecine qui persistèrent bien au-delà du jeu de circonstances de la Libération ${ }^{4}$.

De la Grande-Bretagne aux États-Unis : la pénicilline de guerre

4 Toute bonne histoire de la découverte de la pénicilline débute par une référence au geste de Alexander Fleming examinant une boîte de Pétri oubliée sur un coin de paillasse puis contaminée par un champignon producteur d'une substance capable de diffuser dans le milieu et d'empêcher la croissance de staphylocoques. Pourtant, même s'il obtint pour cela un prix Nobel, sir A. Fleming « n'a pas découvert la pénicilline ${ }^{5}$ ». Ou plutôt, la pénicilline qui occupa longtemps les étagères de son laboratoire n'était pas un médicament. C'était un réactif de laboratoire, utile pour cultiver et sélectionner les micro-organismes, pas un agent thérapeutique.

5 La pénicilline ne commence à changer de statut qu'à la fin des années 1930, lorsqu'un groupe de biochimistes de l'université d'Oxford, alors dirigé par Howard Florey, entreprend de purifier ce que ce dernier voyait comme une enzyme antibactérienne ${ }^{6}$. H. Florey et le biochimiste Ernst Chain obtiennent le soutien de la fondation Rockefeller pour mener à bien cette recherche. La déclaration de guerre modifie les termes du programme. L'urgence scientifique et médicale pousse H. Florey et son groupe à voir dans la purification non le moyen d'étude d'une protéine aux propriétés étonnantes mais une étape dans la préparation d'un agent potentiellement utilisable en médecine humaine ${ }^{7}$.

6 Deux ans plus tard, en juillet 1941, H. Florey et un de ses assistants, George N. Heatley, se rendent aux États-Unis pour démarcher les industriels de la pharmacie afin d'obtenir le lancement d'un programme de production de la pénicilline à grande échelle Imperial Chemical Industry et les firmes anglaises que H. Florey avait d'abord consultées mais en vain. Pourtant, pour les chercheurs d'Oxford, la cause est entendue : la pénicilline est un composé actif et sans danger. Ils en ont pour preuve quelques dizaines de souris qu'ils ont guéries de plusieurs types d'infections, quelques enfants traités avec succès pour des infections oculaires, et une demi-douzaine de patients dont l'état a été temporairement amélioré. L'argument de $\mathrm{H}$. Florey est simple : faute de quantités suffisantes de pénicilline, on ne peut pas poursuivre les essais, guérir des patients en nombre significatif, prouver l'efficacité de la thérapie, en définir les conditions et limites d'usage. La situation ressemble fort à un cercle vicieux. Pour obtenir suffisamment de pénicilline, il faut s'engager dans un programme de développement et de production de masse mobilisant les compétences des industriels de la fermentation et des chimistes. Mais pour qu'une firme s'engage dans un tel programme, il faut que ses dirigeants soient convaincus de la valeur - thérapeutique et 
commerciale - du produit. Or, une telle démonstration exige une quantité importante de pénicilline et donc une initiative industrielle. Du côté des firmes pharmaceutiques, le voyage américain de H. Florey eut des effets mitigés. Les firmes Merck et Squibb ont acquis quelques connaissances et intérêt pour la pénicilline, mais il s'agit de recherches menées « au cas où ». Pour pousser les industriels à voir les choses autrement, il faut l'intervention d'une autorité capable de forcer l'engagement et de construire, autour de la pénicilline, un réseau liant laboratoires, sites de production et hôpitaux.

7 L'Office for Scientific Research and Development (OSRD) de Vannevar Bush joue ce rôle ${ }^{8}$. La transformation de la pénicilline en médicament est donc, parallèlement aux expérimentations des Britanniques, le produit d'une invention organisationnelle : la mise sur pied d'un " programme " fédéral susceptible de " tenir " ensemble des biologistes, des médecins, des chimistes et des ingénieurs afin de pousser la production, de tester les usages du médicament et finalement de le rendre efficace. Jouant de son pouvoir de mettre à disposition des industriels les avancées réalisées par le département de l'agriculture et les laboratoires sous contrat public, le Committee for Medical Research de l'OSRD parvint à constituer un cartel " pénicilline » composé des firmes pharmaceutiques Merck, Squibb et Pfizer'.

Un exemple caractéristique des effets de cet alignement est le développement d'un procédé de culture "en profondeur ». L'objectif est de rendre possible l'abandon des techniques " en surface " utilisées initialement (figure 1). Exigeant en oxygène, le penicillium croît en effet à la surface des milieux de culture. Les Britanniques ont ainsi pris l'habitude d'ensemencer des dizaines, voire des milliers, de fioles. Une culture dans toute la masse du milieu est considérée par les Américains comme préférable et plus économique car praticable dans les grands fermenteurs utilisés pour la culture de bactéries anaérobies et la production d'acides aminés. La mise au point de la technique repose sur la sélection de nouvelles souches, le changement des milieux de culture et la construction d'un nouveau type de fermenteurs. C'est-à-dire sur un ensemble de " petits " changements techniques réalisés dans chacun des laboratoires du nouveau réseau bio-industriel.

9 La recherche de guerre se solde donc par l'émergence de pénicillines distinctes par leurs lieux de fabrication, leurs usages et leurs valeurs symboliques. La pénicilline britannique est pour l'essentiel issue des laboratoires de microbiologie et de biochimie. Secondairement, elle était devenue médicament industriel produit par fermentation en surface. La pénicilline américaine est un enfant de l'OSRD et de la coopération entre industrie, médecine et gouvernement. Fruit de l'ingénierie industrielle, la molécule est désormais une marchandise produite en masse, dans des usines gigantesques, vendue à des prix diminuant très rapidement. De plus, parce que les investissements massifs dans la recherche d'un procédé de synthèse de la molécule n'ont pas (encore) abouti, la pénicilline marque l'avènement d'une nouvelle pratique pharmaceutique centrée sur la manipulation, à grande échelle, des moisissures. Comment les Français vont-ils se saisir de ces diverses pénicillines?

Catching-up ? L'armée et l'Institut Pasteur organisent le Centre Cabanel

Avec la reprise des échanges scientifiques et techniques, en particulier avec l'organisation de missions d'information destinées à révéler aux chercheurs français les fruits de la mobilisation alliée, la pénicilline occupe immédiatement une place de choix dans les réflexions des autorités françaises. à la fin de 1944, deux initiatives sont prises en parallèle. D'une part, le ministère de la Guerre décide, en la personne du général 
Billotte, responsable du service de santé, de créer une commission de la pénicilline. D'autre part, la représentation scientifique française en Grande-Bretagne, dirigée par le biochimiste Louis Rapkine, propose d'organiser une " mission d'information sur la production de pénicilline ». Celle-ci rassemble finalement les deux parties : des chercheurs de l'Institut Pasteur et des pharmaciens militaires. La mission consiste essentiellement en des visites d'usines menées sous la conduite du Ministry of Supply britannique. Officiellement, ce qu'on rapporte tient en quatre points : une liste des souches utilisées, la description du milieu de culture du champignon, les protocoles de dosage de la pénicilline et une comparaison détaillée des systèmes de production de chaque usine.

11 Le retour en France s'accompagne d'une double mobilisation. D'un côté, les capitaines Broch et Dreyfus font pression sur le service des Poudres pour accélérer la mise en place d'un centre de production basé sur l'exploitation rapide des techniques de culture en surface. Implanté dans des locaux mis à disposition par la pharmacie centrale des hôpitaux, celui-ci est situé rue Cabanel. De l'autre, Jacques Tréfouël, directeur de l'Institut Pasteur, insiste sur la nécessité de conduire des recherches et tente de faire de son centre le dépositaire exclusif de cette mission. Fort des premiers contacts avec l'armée, il informe le ministère du lancement d'un projet de recherches à " mener dans le plus grand secret ${ }^{10} »$. Sans doute inspiré par les pratiques de l'OSRD, J. Tréfouël songe alors à une sorte de programme coordonné (" une première tentative de recherches dirigées ») impliquant une bonne partie des services de l'Institut. Fruit de la conjonction entre ces deux initiatives, le Centre Cabanel devient à la fois un site industriel et un site d'application des recherches pastoriennes sur les souches de Penicillium, leurs conditions de culture, les dosages et effets de l'antibiotique. Dans le même temps, les malentendus et tensions entre les différents protagonistes (militaires, biologistes et médecins) du projet de pénicilline française se multiplient.

12 Le premier bilan du Centre Cabanel, rédigé par le pastorien Federico Nitti, en témoigne. Pour le chercheur, l'Institut Pasteur a fait l'essentiel du travail et apporté « ses scientifiques, ses fournisseurs, servi de caution, mis les militaires en rapport avec Pierre Grillet de Rhône-Poulenc pour l'extraction ${ }^{11} »$. Malheureusement, sur place, leur autorité est sans cesse contestée : «P. Broch essaie de devenir autonome [...] le Centre comprend quatre capitaines et de nombreux gradés mais seul le capitaine Netik s'est un peu intéressé aux problèmes touchant la pénicilline, les officiers du Centre ne possèdent aucune notion de bactériologie industrielle. » à court terme, F. Nitti souhaite que l'on dise clairement qu'il ne dépend pas de l'autorité de P. Broch. De leur côté, les médecins militaires manifestent peu d'intérêt pour les raffinements scientifiques de $\mathrm{F}$. Nitti et veulent que l'on aille le plus vite possible vers l'obtention de quantités significatives de pénicilline. Sans fourniture rapide au service de santé des armées, le Centre Cabanel risque d'être perçu comme inutile et, à terme, menacé.

13 Les archives que nous avons pu consulter ne livrent aucune indication sur la quantité de pénicilline obtenue au Centre Cabanel. En tout cas, durant l'hiver 1945-1946, l'image $\mathrm{du}$ Centre auprès du ministère de la Guerre est suffisamment bonne pour que ce dernier et le gouvernement provisoire envisagent de l'étendre. L'initiative la plus spectaculaire est d'ordre parlementaire. Au début de 1946, commence à circuler un projet de loi portant création d'un Office national industriel des produits biochimiques $(\mathrm{ONIPB})^{12}$. Cet office coifferait le Centre Cabanel et les usines de production de masse 
qu'aussi bien les experts du corps des Poudres que le ministère de la Santé imaginaient (chacun de son côté) construire ${ }^{13}$.

Inspiré du statut de l'office de l'azote créé dans les années 1930 pour suppléer aux carences de l'industrie chimique, le projet ne dépasse pas le stade du brouillon institutionnel. Il reste toutefois emblématique d'une certaine vision des rapports entre État, science et industrie. Élaboré dans un contexte politique marqué par les nationalisations et d'une façon générale par l'idée d'un État fort responsable du développement économique et social du pays, le projet d'ONIPB fait très directement écho à la vision des services publics nationaux défendue par le Conseil national de la résistance, et plus spécifiquement par l'alliance qui unit pour un temps grands corps et " compagnons de route " du parti communiste ${ }^{14}$. L'office des produits biochimiques est en effet un véritable hybride entre un service technique de l'armée, une usine nationale et une agence étatique de recherche. Par là, il entretient quelques parentés avec un CEA au sort nettement plus heureux ${ }^{15}$.

15 Le préambule motivant la création d'un tel office renvoit, contexte industriel oblige, à la saga américaine de la pénicilline. Indépendance nationale et santé publique sont les deux raisons ensuite invoquées pour justifier la création d'une structure publique de production : « La production en France de ce médicament ne saurait laisser indifférents les pouvoirs publics. Il importe en effet que l'État ait entre les mains un moyen d'assurer un contrôle efficace de ce produit qui présente une si grande importance en santé publique. Aussi sans vouloir créer un monopole, l'État doit-il créer un office national qui s'occupera des recherches et de la fabrication de la pénicilline tout en laissant subsister en face de cet office des sociétés privées qui se seraient mises à l'étude du même problème mais sans demander le secours de l'État. »

Instrument de la puissance publique, faisant de fait concurrence à l'industrie pharmaceutique privée, l'office ne peut espérer réussir à s'attribuer une part importante de la production d'antibiotiques que parce que le cas de la pénicilline est très particulier. Pour la suite, les rédacteurs du projet de loi envisagent en effet le développement de toute une gamme de produits antibiotiques et non de la seule pénicilline. Selon eux, l'office devrait miser sur la recherche et sur les liens avec les laboratoires publics : «L'office [...] formera les équipes nécessaires tant à la recherche qu'à la fabrication. Les techniciens de cet office travailleront en liaison avec le CNRS et l'Institut Pasteur, les usines de l'office peuvent être considérées comme des usines d'application. Il convient de noter, en effet, que les chercheurs de nos instituts et de nos facultés n'ont généralement pas les moyens de faire expérimenter à l'échelle semiindustrielle et à l'échelle industrielle les procédés qu'ils ont mis au point dans leurs laboratoires. [...] Au début de ses travaux, l'office fera appel aux brevets étrangers et notamment aux brevets américains étant donné l'avance que possèdent les sociétés américaines dans la fabrication de la pénicilline. Pour l'avenir, l'office devra étudier et mettre au point ses propres procédés de fabrication [...], sa principale mission étant de généraliser le plus possible l'emploi des nouveaux produits biochimiques dont la médecine moderne a su démontrer les bienfaits. "Peuplé d'ingénieurs des corps techniques de l'État, l'office serait une structure administrée par un conseil représentant " les ministères intéressés, les savants, les chercheurs du CNRS, des facultés, et le personnel de l'office ».

17 Cette proposition est l'une des mises en forme les plus élaborées de ce que l'on peut tenir pour un modèle français de mobilisation de la recherche biologique pour le 
développement technologique et la production industrielle. Dans ce cadre, l'État est appelé à jouer un rôle central non seulement comme puissance coordinatrice (ce qui était le cas pour la recherche de guerre américaine) mais aussi comme inventeur, entrepreneur, payeur et consommateur des nouveaux médicaments. Le projet d'office peut être vu comme un écho tardif au système de relations entre savants, administrateurs, utilisateurs que les militaires ont déjà expérimenté, que ce soit dans les usines d'armement contrôlées par les Poudres ou dans les services techniques de la Marine où cohabitaient ingénieurs des grands corps et savants des facultés ou du $\mathrm{CNRS}^{16}$.

Emblématique de l'état d'esprit régnant tout de suite après la Libération, cette proposition est devenue totalement incongrue dès la fin de l'année 1946 alors que les rapports entre l'État et l'industrie privée se "normalisent ». Surtout si, comme la rhétorique qui l'irrigue permet de le soupçonner, le projet d'ONIPB procède de sources proches du PCF ${ }^{17}$.

Quel que soit le sort de ce projet d'ONIPB, la seconde moitié de l'année 1945 et le début de l'année 1946 correspondent à la période d'apogée du Centre Cabanel. Les discussions sur la construction de grandes usines de production de pénicilline basées sur l'expérience parisienne deviennent plus concrètes. À la fin de 1945, le service des Poudres et le ministère de l'Armement appuient l'idée de construire très vite une grande usine de fermentation en surface dans le nord de la France, au Ripault ${ }^{18}$. Les pastoriens s'opposent radicalement à cette perspective.

20 Pour les militaires et l'administration, un objectif prioritaire est d'éviter autant que faire se peut les importations de pénicilline. La production en surface est vue comme le moyen de fournir rapidement les services de santé ${ }^{19}$. Rencontrant les représentants des Poudres le 9 février 1946 pour tenter de résoudre une nouvelle fois les tensions avec les officiers du service de santé, J. Tréfouël et $\mathrm{F}$. Nitti rétorquent que la solution d'une usine de production en surface n'est pas " recommandable » dans la mesure où elle repose sur des bases techniques déjà obsolètes. Pour eux, il faut se rallier à la technique américaine de fermentation en profondeur (figure 2) et s'en remettre à l'expérience des opérateurs privés, les firmes Rhône-Poulenc et Roussel. " Les industriels qui ont déjà étudié la question sont les mieux placés pour produire rapidement de la pénicilline car ils sont déjà depuis longtemps en rapport avec les Américains, ils ont des spécialistes formés qui ont vu en Amérique les installations en cuve et obtiendront les appareils très rapidement par leurs relations avec les firmes américaines. ${ }^{20}$ » Le rôle du Centre Cabanel devrait donc être, au moins dans le cas de la pénicilline, d'aider au développement des capacités de l'industrie pharmaceutique en mettant au point une " méthode française de procédé en cuve ", un des rares aspects de la production de pénicilline sur lequel les brevets américains sont difficiles à contourner ${ }^{21}$.

On en reste là. Les Poudres continuent à planifier une installation en surface dans la poudrerie du Ripault, tandis que la recherche sur la fermentation en profondeur se poursuit dans un Centre Cabanel réorganisé selon les vœux de F. Nitti et J. Tréfouël. Désormais, les pastoriens assument seuls la direction scientifique, les officiers du service de santé ayant été remplacés par des biologistes. Les négociations sont indirectement relancées par le CNRS. Celui-ci, sur l'initiative du biologiste Émile Terroine, alors soucieux de ne pas délaisser un secteur aussi important, a mis en place sa propre commission de la pénicilline, laquelle regroupe non seulement les militaires (le colonel Fauveau, les commandants Broch et Bouissou, le capitaine Netik) mais aussi 
les biochimistes les plus renommés du centre (Claude Fromageot, René Wurmser, Eugène Aubel, Pierre Lemoigne, André Lwoff).

Une des missions initiales de la commission est de préparer la mise en œuvre de l'ONIPB. En juin 1946, une délégation composée de P. Broch, Fauveau, C. Fromageot et A. Lwoff est envoyée en Grande-Bretagne pour y évaluer les techniques de fabrication de la pénicilline par fermentation en profondeur ${ }^{22}$. Très vite mis à la disposition de la commission, le rapport de la délégation n'est signé que des biochimistes ${ }^{23}$. Le document insiste sur l'efficacité des fermentations en profondeur et sur les augmentations de rendement dues à deux développements : le changement de taille des cuves qui atteignent désormais 20 à 40000 litres, le choix d'une autre espèce de penicillium issue de la sélection de souches dites "à haut rendement ". De plus, les deux chercheurs rapportent que, pour les Britanniques eux-mêmes, la fermentation en surface est condamnée. La compagnie Wellcome est désormais sur le point de fermer ses installations de ce type. Face à la modernité américano-britannique, les conclusions semblent évidentes.

23 La réunion de la commission est prévue pour le 4 juillet 1946. Le cabinet du ministère de l'Armement, soucieux de ménager un compromis avec les scientifiques, a finalement proposé que deux usines, une en surface et une en profondeur, soient réalisées simultanément. Dans le cours de la discussion, le commandant Bouissou, représentant des Poudres, laisse toutefois clairement entendre que la construction des deux usines n'est pas possible et qu'à court terme, il n'engagera la construction que de la seule usine de production en surface ${ }^{24}$. Les biochimistes décident alors de préparer un avis exprimant leur opposition à cette manifestation d'autoritarisme militaire. Rédigé par é. Terroine et destiné au directeur du CNRS, ce texte stipule que "le gouvernement français ne doit sous aucun prétexte s'engager dans une nouvelle production faisant appel aux procédés de culture en surface. Ces procédés sont en effet partout abandonnés ». Pour les chercheurs, le projet militaire de production nationale est techniquement rétrograde et économiquement déraisonnable. Faut-il en conclure que les Poudres et plus généralement l'administration doivent cesser de s'occuper de produire la pénicilline ? La réponse est négative : " Il y a lieu de procéder immédiatement à la réalisation d'une usine de fabrication de pénicilline en profondeur. "Scientifiquement plus intéressante, une telle usine implique le perfectionnement des techniques de fermentation, la poursuite des recherches : «Il est de toute nécessité de prévoir dans la société des produits biochimiques de nouveaux laboratoires tendant : a) à préciser les méthodes de contrôle thérapeutique des antibiotiques [...], les procédés de dosage actuellement en usage ne donnant pas une indication suffisante sur la valeur thérapeutique des produits obtenus ; b) à poursuivre des recherches dans tout le domaine des antibiotiques autres que la pénicilline afin d'être prêt, le cas échéant, soit à substituer les fabrications de ces produits à la pénicilline, soit à les y ajouter. ${ }^{25}$ » De plus, il faut poursuivre les missions en Grande-Bretagne (figure 3) : « La mise au point de l'usine pourrait être grandement facilitée par la connaissance des dispositifs de l'usine nationale anglaise de Liverpool dont il serait vraisemblablement possible d'obtenir l'autorisation de visite. "

Le texte finalement adopté reprend l'essentiel de l'argumentation contre la construction d'une seconde installation de production en surface ${ }^{26}$. La discussion sur le passage à la production de masse et les enjeux industriels a donc reconfiguré les alliances. Un bloc scientifique associant les chercheurs du CNRS et de l'Institut Pasteur 
s'oppose désormais aux techniciens des Poudres et du service de santé des armées. Les premiers jouent de la modernité technique américaine pour faire avancer l'idée d'un centre des antibiotiques tourné vers une recherche pérenne visant en particulier la mise au point de nouveaux médicaments biologiques. à court terme, cette perspective revient à délaisser la pénicilline pour investir le développement de la streptomycine, nouvel antibiotique dont on vient d'apprendre la découverte aux États-Unis. Les seconds entretiennent l'idée de production nationale en arguant de l'urgence des besoins et de l'adaptabilité de la technique anglaise.

L'orientation scientifique semble avoir prévalu puisque, dès le début de l'année 1947, le Centre Cabanel a cessé toute activité de production de pénicilline pour se transformer en centre d'investigation sur les nouveaux antibiotiques. Paradoxalement, cette victoire ne correspond pas à l'adoption des plans de l'Institut ou du CNRS, lesquels incluaient la mise au point de dispositifs " français » de fermentation en profondeur mais venaient sanctionner les changements du marché des antibiotiques qui rendirent la question de la production étatique obsolète. à partir de la fin de l'année 1946 (et le mouvement ne fit que s'amplifier en 1947), les prix de la pénicilline américaine chutent rapidement tandis que les quantités disponibles semblent désormais illimitées ${ }^{27}$. Les importations effectuées par la Direction centrale de la pharmacie se multiplient ${ }^{28}$. à l'été 1946, Paris Médical note : "Le ministère de la Santé publique communique une augmentation considérable des importations, auxquelles s'ajoutera prochainement l'apport de la production française, qui a permis d'apporter un assouplissement notable des conditions d'utilisation de la pénicilline. " Parallèlement aux importations, les firmes Rhône-Poulenc et Roussel ont acheté des licences pour les techniques de fermentation à grande échelle et démarrent leur propre production en profondeur ${ }^{29}$. La prédiction de É. Terroine se réalise à une vitesse telle que les utilisateurs de pénicilline - services de santé militaire compris - se trouvent en position de pouvoir satisfaire les besoins les plus urgents sans même qu'une production d'État significative n'ait démarré. à la fin de 1946, les quantités disponibles sont suffisantes pour que les mesures de répartition soit levées et que l'on puisse revenir à l'organisation classique du marché des médicaments : tout pharmacien disposant d'un frigidaire pouvait désormais commander et vendre la pénicilline ${ }^{30}$.

Progressivement, malgré l'achèvement de l'usine du Ripault, les Poudres se désengagent de la production d'antibiotiques, laissant le Centre Cabanel suivre une carrière modeste de site de recherche « biotechnologique » associé d'abord à l'Institut Pasteur puis au Centre de transfusion sanguine. Dans le courant de l'année 1947, on s'y intéresse aux nouveaux antibiotiques (gramicidine, streptomycine et autres candidats au sort moins brillant) et aux conditions de leur production. Au début de 1948, les Poudres se retirent définitivement de l'entreprise. La direction de l'Institut Pasteur réagit en recentrant les recherches sur son propre site et sur les aspects plus fondamentaux de la biologie des antibiotiques. Les laboratoires de biochimie (Marcel Machebœuf) ou des fermentations (P. Lemoigne) continuent donc à s'occuper du mode d'action de la pénicilline, des problèmes de résistance acquise par les bactéries, de la structure et de voies de synthèse de la molécule. Les activités " antibiotiques » du Centre Cabanel cessent. Les biologistes et pharmaciens qui y travaillent doivent se reconvertir. Fort des compétences acquises en matière de production, G. Netik participe par exemple à la création du Laboratoire français des antibiotiques et débute une carrière de cadre de l'industrie pharmaceutique. 
L'alliance entre savants et militaires se solde donc par un double abandon : celui d'une recherche directement au contact de la production et celui d'un service public des produits biologiques calqué sur le modèle d'EDF ou du CEA et contrôlé par les grands corps techniques de l'État.

Conclusion

L'histoire du développement de la pénicilline durant la guerre a été interprétée de diverses manières. On a pu la lire comme une illustration de la nécessité de développer la recherche biologique "fondamentale " pour constituer un réservoir de connaissances ensuite applicable à des besoins très variés. On a pu aussi la présenter comme un exemple d'innovation technique, un témoignage éloquent de la fécondité des collaborations entre industriels de la pharmacie et cliniciens. Les deux lectures ne sont pas antinomiques. L'ancien responsable de l'OSRD, V. Bush, les a par exemple associées dans son ouvrage intitulé Science: The Endless Frontier, dans lequel il dessine les traits de ce que devrait être, selon lui, la future politique scientifique du gouvernement fédéral américain. Porteuse de multiples significations, la pénicilline est devenue un symbole du nouveau système d'échanges et de collaborations liant les laboratoires universitaires de biologie, les grands hôpitaux, les divisions de recherche de l'industrie, et les agences de recherche étatiques. Après 1945, ce " complexe biomédical » est particulièrement visible et dense aux États-Unis. Il existe aussi en France, mais avec des formes spécifiques.

29 Parmi ces dernières, on a souvent parlé de la faiblesse de la recherche industrielle. On considère ainsi que les liens entre laboratoires publics et industriels sont restés ténus, que l'État est à l'origine de l'essentiel des activités de recherche et développement. Plus spécifiquement, dans les années d'après guerre l'industrie pharmaceutique a privilégié l'importation de savoir-faire et les achats de licence, plutôt que la constitution de sa propre base de connaissances. L'épisode de l'introduction de la pénicilline confirme certains aspects de ce jugement : par exemple, la juxtaposition conflictuelle des logiques d'action respectives des biologistes, des militaires et des industriels de la pharmacie. La collaboration au Centre Cabanel apparaît ainsi comme le produit d'un moment tout à fait particulier dans lequel on peut voir l'empreinte de la tradition d'intervention des grands corps techniques liés aux militaires, les effets de la mobilisation militaire de 1944-1945, la prégnance d'un climat politique favorable aux nationalisations et aux grandes réformes de l'État.

L'histoire de l'introduction de la pénicilline éclaire aussi les limites d'une grille de lecture faisant des biotechnologies un problème permanent des dispositifs français de recherche. L'épisode du Centre Cabanel révèle en effet l'existence d'un modèle particulier de recherche biotechnologique. Centré sur les relations étroites qui lient l'Institut Pasteur à l'État, celui-ci reposait sur une délégation d'expertise contournant l'industrie chimique et pharmaceutique. Institution de droit privé, l'Institut associait en effet étroitement expérimentation de laboratoire, découverte et production de nouveaux produits biologiques à usage médical, mise à disposition de ces biens sur un quasi-marché. Dans la mesure où une part importante des coûts était, au nom des impératifs de santé publique, couverte par les subventions des administrations, on peut voir dans cette combinaison une délégation de ce qui aurait pu être, sur le modèle de l'ONIPB, l'activité d'un corps technique d'État. Loin d'être une survivance du règne des savants inventeurs et entrepreneurs de la fin du XIX ${ }^{\mathrm{e}}$ siècle, ce modèle est, pendant une 
bonne partie de l'après-guerre, resté le cadre de mise au point de nombreux produits biotechnologiques, à commencer par le vaccin contre la poliomyélite.

\section{NOTES}

1.J.-F. Picard, La République des savants. La recherche française et le CNRS, Flammarion, Paris, 1990. D. Dosso, « Louis Rapkine et la mobilisation scientifique de la France libre ", thèse, université Paris VII, 1998.

2.J.-F. Picard, « Poussée scientifique ou demande de médecins ? La recherche médicale en France de l'Institut national d'hygiène à l'INSERM », Sciences sociales et santé, 1992, 10 (4), p. 47-106.

3.J.-P. Gaudillière, Inventer la biomédecine. La France, l'Amérique et la production des savoirs du vivant après 1945, La Découverte, Paris, 2002.

4.Cet article s'appuie sur le chapitre premier de J.-P. Gaudillière, Inventer la biomédecine..., op. cit. Pour une analyse complémentaire de l'histoire française de la pénicilline, en particulier des stratégies industrielles, voir V. Quirke, Experiments in Collaboration. The Changing Relationship between Scientists and Pharmaceutical Companies in Britain and France, 1935-1965, Oxford University, 1999.

5.G. Macfarlane, Alexander Fleming. The Man and the Myth, Harvard University Press, 1984 ; Chen, Pourquoi Fleming n'a pas découvert la pénicilline, Institut Sanofi-Synthélabo, Les Empêcheurs de penser en rond, Paris, 1997.

6.W. Trevor, Howard Florey. Penicillin and After, Oxford University Press, 1984.

7.Sur l'urgence de l'automne 1940 et la recherche médicale, voir l'exemple du vaccin contre la grippe de la fondation Rockefeller massivement acheté par le ministère de la Santé publique ; J.-P. Gaudillière, "Rockefeller Strategies for Scientific Medicine: Molecular Vaccines, Viruses, and Vaccines ", Studies in History and Philosophy of the Biological and Biomedical Sciences, 31, 2000, p. 491-509.

8.G. L. Hobby, Penicillin: Meeting the Challenge, Yale University Press, 1985.

9.P. Neushul, "Science, government and the mass production of penicillin ", Journal of the History of Medicine and Allied Sciences, 1993, 48, p. 371-395.

10.Lettre de J. Tréfouël au ministre de la Guerre, 22 février 1945, AIP, DIR SER 49, chemise « Mise en place du Centre Cabanel ».

11.AIP, DIR SER 49, chemise « Mise en place du Centre Cabanel », F. Nitti à J. Tréfouël, 19 mars 1945.

12.Projet de loi portant création de l'ONIPB, sans date, AIP, DIR SER 49, chemise « Pénicilline divers ».

13.Les informations dont nous disposons sur le projet d'office proviennent des seules archives de l'Institut Pasteur et du ministère de la Santé. Nous n'avons pas pu retrouver qui était à l'origine de la proposition de loi.

14.Sur la politique industrielle de la Libération, voir C. Andrieu, L. Le Van et A. Prost, Les Nationalisations de la Libération, Presses de la FNSP, Paris, 1987. P. Mioche, Le Plan Monnet. Genèse et élaboration, 1941-1947, Publications de la Sorbonne, Paris, 1987. 
15.Sur l'histoire du CEA, F. Jacq, Pratiques scientifiques, formes d'organisation et représentations politiques de la science dans la France d'après guerre, École nationale supérieure des Mines de Paris, 1996.

16.Sur ce modèle, voir F. Jacq, Pratiques scientifiques..., op. cit.

17.Un indice en ce sens est l'implication du CNRS alors dirigé par G. Teissier dans la préparation d'une infrastructure scientifique pour l'ONIPB, avec la formation d'un comité ad hoc en charge du suivi du dossier pénicilline. Voir ci-après.

18.Entretien du 12 mars 1946 entre J. Tréfouël, F. Nitti, Fauveau (direction des Poudres), Le Queinec (directeur de cabinet du ministre de l'Armement), IAP DIR SER 49, chemise « Mise en place du Centre Cabanel ».

19.Entretien J. Tréfouël, F. Nitti, Fauveau, du 9 février 1946, AIP, DIR SER 49.

20.Ibid.

21.G. Hobby, Penicillin..., op. cit.

22.Rapport de C. Fromageot et A. Lwoff, AIP, DIR SER 49, chemise « Pénicilline CNRS ».

23.Aucun document ne témoigne du désaccord des militaires même si la suite des événements laisse supposer qu'ils pouvaient être moins affirmatifs.

24.Procès-verbal de réunion de la commission du CNRS, 4 juillet 1946, AIP, DIR SER 49, chemise « Pénicilline CNRS ».

25.Note signée É. Terroine, AIP, DIR SER 49, chemise « Pénicilline CNRS ».

26.Vœu de la commission CNRS, AIP, DIR SER 49, chemise « Pénicilline CNRS ».

27.Sur la chute des prix et la croissance des quantités, voir Hobby, Penicillin..., op. cit.

28. « Les conditions d'utilisation de la pénicilline », Paris Médical, juillet 1946.

29. «Échos et nouvelles. Fabrication française de la pénicilline ", Strasbourg médical, février 1947, p. 34. Sur la production de pénicilline à Rhône-Poulenc, voir V. Quirke, Experiments in Collaboration..., op. cit.

30.« Note sur la distribution de pénicilline », Paris Médical, octobre 1946.

\section{INDEX}

Mots-clés : biologie, biologiste, pénicilline, Institut Pasteur, centre Cabanel, fermentation, streptomycine, antibiotique, Rhône-Poulenc, Roussel, poliomyélite

\section{AUTEUR}

\section{JEAN-PAUL GAUDILLIÈRE}

Jean-Paul Gaudillière, chargé de recherche (Inserm), est membre du Centre de recherche sur la médecine, les sciences, la santé et la société (Cermes, Inserm-CNRS). 